\title{
ZOMBIE VORTEX INSTABILITY IN THE PROTOPLANETARY DISK: CAN WE FIND IT IN THE LAB?
}

\author{
G. Facchini ${ }^{1,2}$, M. Wang ${ }^{2}$, P. Marcus ${ }^{2}$ and M. Le Bars ${ }^{1}$
}

\begin{abstract}
Without instabilities, the gas in the protoplanetary disk approximately a forming protostar remains in orbit rather than falling onto the protostar and completing its formation into a star. Moreover without instabilities in the fluid flow of the gas, the dust grains within the disk's gas cannot accumulate, agglomerate, and form planets. Keplerian disks are linearly stable by Rayleigh theorem because the angular momentum of the disk increases with increasing radius. This has led to the belief that there exists a large region in protoplanetary disks, known as the dead zone, which is stable to pure hydrodynamic disturbances. The dead zone is also believed to be stable against magneto-rotational instability (MRI) because the disks' cool temperatures inhibit ionization and therefore prevent the MRI. A recent study Marcus et al. (2013) shows the existence of a new hydrodynamic instability called the Zombie Vortex Instability (ZVI), where successive generations of self-replicating vortices (zombie vortices) fill the disk with turbulence and destabilize it. The instability is triggered by finite-amplitude perturbations, including weak Kolmogorov noise, in stratified flows with Brunt-Väisälä frequency $N$, background rotation $\Omega$ and horizontal shear $\sigma$. So far there is no observational evidence of the Zombie Vortex Instability and there are very few laboratory experiments of stratified plane Couette flow with background rotation in the literature. We perform systematic simulations to determine where the Zombie Vortex Instability exists in terms of the control parameters (Reynolds number $R e, \sigma / f$ and $N / f$ ). We present a parameter map showing two regimes where ZVI occurs, and interpret the physics that determines the boundaries of the two regimes. We also discuss the effects of viscosity and the existence of a threshold for Re. Our study on viscous effects, parameter map and its underlying! physics provide guidance for designing practical laboratory experiments in which ZVI could be observed.
\end{abstract}

${ }^{1}$ Aix-Marseille Univ., CNRS, Centrale Marseille, IRPHE, Marseille, France

${ }^{2}$ Department of Mechanical Engineering, University of California, Berkeley 6121 Etcheverry Hall, Mailstop 1740, Berkeley, CA 94720-1740, USA 


\section{Introduction}

The stability of a rotating flow with vertical stratification and horizontal shear has drawn great attention in recent years, due to the the interest of astrophysicists in determining a mechanism that can create turbulence in a protoplanetary accretion disk. This topic is of particular interest in the regions of a nearly Keplerian protoplanetary disk (PPD) where gas is weakly ionized, because the magneto-rotational instability MRI (Chandrasekhar 1960; Hawley \& Balbus 1991) cannot occur, motivating the appellation of dead zones for such a regions. In unstratified Keplerian disks, we expect the flow to be stable to purely hydrodynamic instabilities because the angular momentum increases with increasing radius, i.e. it is stable by Rayleighs criterion (Rayleigh 1917). Similarly, in unstratified Taylor-Couette (TC) flows in which the angular momentum increases with radius, we also expect the flow to be stable. Nevertheless if the fluid is stratified, such a configuration may become unstable (Molemaker et al. 2001; Yavneh et al. 2001). The possible existence of this new linear instability in weakly ionized disks was analysed by Dubrulle et al. (2005) who also introduced the appellation of Strato-RotationalInstability SRI. Further work on SRI was performed by Shalybkov \& Rüdiger Shalybkov \& Rüdiger (2005) whose theoretical predictions were confirmed in the first experimental observation of SRI, reported by Le Bars \& Le Gal (2007). More recently Lesur et al. (2014) and Gole et al. (2016) studied the effect of a MRIdriven inner core on an outer ohmic dead zone, while Nelson et al. (2013) and Barker \& Latter (2015) studied the Vertical Shear Instability, possibly induced by the thermal or entropic radial structure of accretion disks. For a background scenario similar to SRI, i.e. when rotation, vertical stratification and horizontal shear are of the same order of magnitude, Barranco \& Marcus (2005) first observed the occurrence of a new finite-amplitude instability subsequently analyzed and identified as the self-replicating "Zombie" Vortex Instability ZVI by Marcus et al. (2013). As ZVI evolves, an initial ab initio perturbation in the form of a vortex or wave excites a baroclinic critical layer at a proscribed distance from the perturbation; by vortex stretching and tilting, the critical layer turns into a vortex layer; by linear instability, the vortex layer then rolls up into one or more vortices; these new vortices then excite new critical layers, and the process self-replicates until the entire domain fills with vortices and the flow becomes turbulent. Marcus et al. (2015) confirmed the existence of the instability using more general initial conditions (Kolmogorov noise), and described the late stage instability as a truly turbulent scenario, thus designating ZVI as a favorite candidate to destabilize the dead zone of a protoplanetary disk. Even more recently ZVI was independently recovered by the theoretical analysis of Umurhan et al. (2016) and the numerical simulations of Lesur \& Latter (2016). Conclusions on the prevalence of ZVI in protoplanetary disks remain however under discussion.

In the present work we systematically explore the existence of ZVI in the parameter space of the two dimensionless parameters $\sigma / f$ and $N / f$, where $f$ is the Coriolis parameter (rotation), $N$ is the Brunt-Väisälä frequency (stratification) and $\sigma$ is the cross-stream velocity gradient (shear). We observe that the instability 
region is restricted to a relatively small region for both cyclonic $(\sigma / f>0)$ and anticyclonic $(\sigma / f<0)$ regimes, with the exception of the $N / \sigma \sim 1, f \rightarrow 0$ area, where no boundary could be detected. Preliminary results are also shown for two benchmark cases when viscosity is included in the calculations. In Section 2 we describe the equations we solve and the numerical methods. In Section 3 we review the main features of ZVI, explain how to recognize it, and present the stability diagram showing where the flow is unstable and forms zombie turbulence. In Section 4 we discuss about the regions of the 2-dimensional parameter space that are suitable for experimental investigation, and we comment on the possible coexistence of ZVI and SRI.

\section{Problem set-up}

\subsection{Theoretical frame}

We consider the Navier-Stokes equations for a rotating, linearly stratified and horizontally sheared flow in the Boussinesq approximation. The density gradient is parallel to the rotation axis and aligned with the vertical direction $\hat{z}$, while shear acts along $\hat{\boldsymbol{y}}$.

$$
\begin{gathered}
\frac{\partial \boldsymbol{u}}{\partial t}+(\boldsymbol{u} \cdot \nabla) \boldsymbol{u}=\nu \nabla^{2} \mathbf{u}-\frac{\nabla p}{\rho_{0}}-\frac{\tilde{\rho}}{\rho_{0}} g \hat{\boldsymbol{z}}+f \mathbf{u} \times \hat{\mathbf{z}}-U(y) \frac{\partial \mathbf{u}}{\partial x}+\sigma u_{y} \hat{\mathbf{x}} \\
\frac{\partial \tilde{\rho}}{\partial t}+(\mathbf{u} \cdot \nabla) \tilde{\rho}=\rho_{0} \frac{N^{2}}{g} u_{z}-U \frac{\partial \tilde{\rho}}{\partial x} \\
\nabla \cdot \mathbf{u}=0
\end{gathered}
$$

where $\hat{\boldsymbol{x}}$ is the stream-wise direction, $\hat{\boldsymbol{y}}$ is the cross-stream direction and $\hat{\boldsymbol{z}}$ is the vertical direction. Also we decompose the velocity field as $\mathbf{u}_{\text {total }}=U(y) \hat{\mathbf{x}}+$ $\mathbf{u}(x, y, z, t)$ where $U(y)=-\sigma y$ is the base shear velocity, and the density field as $\rho(x, y, z, t)=\tilde{\rho}(x, y, z, t)+\bar{\rho}(z)$ where $\bar{\rho}(z)=\rho_{0}\left(1-N^{2} z / g\right)$ is the unperturbed density profile.

\subsection{Numerical methods}

The system above is implemented in a periodic cubic domain and the equations solved with a pseudo-spectral method. This approach imposes the use of shearing sheet coordinates $\left(x^{\prime}, y^{\prime}, z^{\prime}, t^{\prime}\right)=(x+\sigma y t, y, z, t)$ to make Equation (2.1) autonomous in $y$. The main part of the present work is done in the inviscid limit (same as Marcus et al. 2013), which corresponds to neglect the first term on the right-hand side of (2.1). To solve numerically the initial value Equations (2.1)-(2.3) we replace viscosity with hyper-viscosity, i.e. $\nu \nabla^{2} \rightarrow \nu_{8}\left(\nabla^{2}\right)^{4}$ in order to prevent the accumulation of energy at the grid scale (we also added a hyper-diffusivity to the density Equation (2.2). One may question whether the hyper-dissipations affect the onset of the instability. We ruled out this possibility by demonstrating that different functional forms of hyper-dissipations lead to the same flow, as 
a
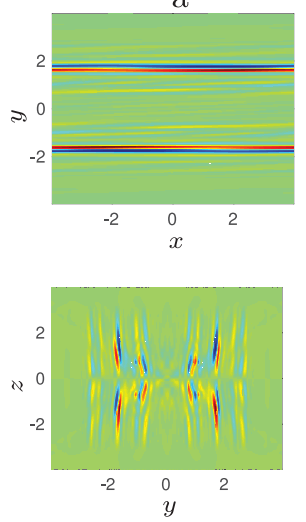

$\mathrm{b}$
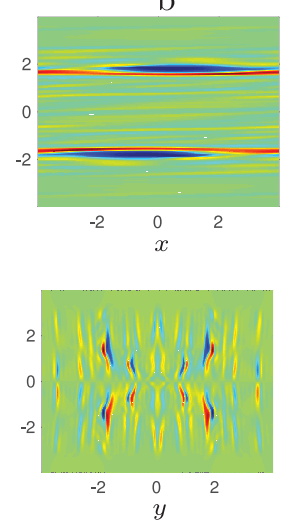

$\mathrm{c}$
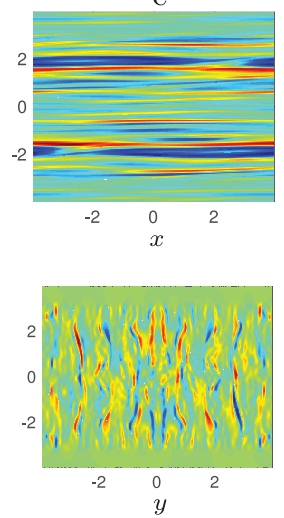

d
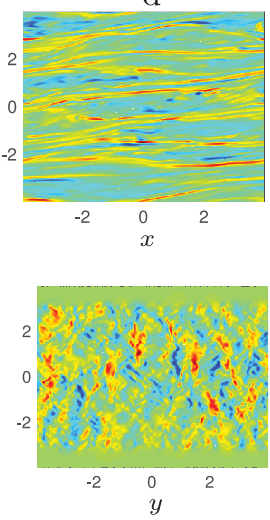

Fig. 1. Vertical vorticity $\omega_{z}$ in the plane $z=1.5(t o p)$ and vertical velocity $u_{z}$ in the plane $x=0$ (bottom) at four different times: $290 / \sigma, 480 / \sigma, 1920 / \sigma, 3840 / \sigma$. Red corresponds to positive values of vorticity and velocity, blue to negative ones (respectively cyclonic and anti-cyclonic flows in the case of vorticity).

long as hyper-dissipation only affects wave numbers that are bigger than the wave numbers that are associated with the baroclinic critical layer. Details about the numerical methods we used and their implementation can be found in Barranco \& Marcus (2006).

\section{Results}

\subsection{ZVI mechanism}

Here we recall briefly the fundamental stages that the flow undergoes when initialized with a $3 \mathrm{D}$ gaussian vortex at the center $(x=y=z=0)$ of the cubic domain $\left(L_{x}=L_{y}=L_{z}=8\right)$. We also provide a convenient tool to detect the onset of ZVI. In Figure 1 (top) we report the vertical vorticity $\omega_{z}$ at four different times in the horizontal plane $z=1.5$, i.e. where the first generation of vortices is created. In Figure 1 (bottom) we report the corresponding evolution of the vertical velocity $u_{z}$ in the vertical mid plane $x=0$. In both sequences we label each panel with the letters $a, b, c, d$, which refer to the four distinct stages we identify. Monitoring $\omega_{z}$ is the most suitable tool to describe the flow throughout all its evolution: the flow is initiated with a single vortex, then a large number of new vortices are created when ZVI is triggered, and late turbulence stage still contains large coherent vorticity structures. The field $u_{z}$ provides an intelligible insight in the instability mechanism, because the vertical velocity is initially null everywhere, and the critical layers characteristic of ZVI appear spontaneously in the form of strong jets or shear layers in this component. However we stress that the critical layers structure appear (not shown here) in all the three components of both $u_{z}$ 

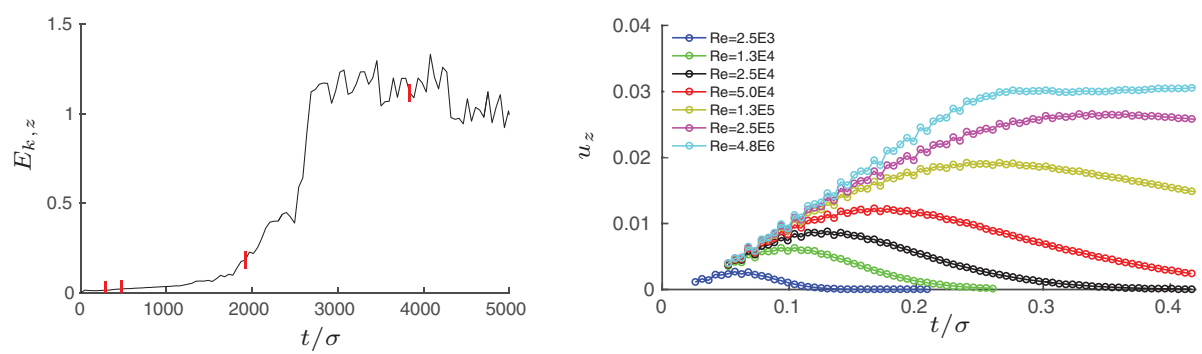

Fig. 2. Left: vertical kinetic energy $E_{k, z}$ as a function of time. Red ticks refer to the sequences in Figure 1. Right: peak of the vertical velocity $u_{z}$ inside the critical layers as a function of time, for different Reynolds numbers (different colours). Note that only the cyan curve appears not decreasing in the end; this is consistent with the fact that it corresponds to the only experiment where $R e \gtrsim R e_{c}$.

and $\omega_{z}$. Simultaneously we also consider the time evolution of the vertical kinetic energy $E_{k, z}$ (Fig. 2), which evolves from zero to almost a constant, and turns out to serve as the most succinct and practical tool to determine whether the flow has created self-sustaining zombie turbulence. All the figures refer to the benchmark case $\sigma / f=-0.75, N / f=1$, but similar features were observed for all unstable cases. Below we schematically describe the generic features of each step:

a) vortex sheet: the first generation of critical layers (Fig. 1 bottom) is visible at the position predicted by Marcus et al. (2013). $\omega_{z}$ field (Fig. 1 top) shows the formation of two pairs of vortex sheets of opposite vorticity (stripes corresponding to planes in the $x-z$ projection), which progressively cover the whole stream-wise direction. $E_{k, z}$ slowly increases (Fig. 2).

b) zombie vortex: Critical layers increase in strength and depending on the anti-cyclonic (cyclonic) nature of the flow, the anti-cyclonic (cyclonic) sheets become unstable and roll up to form the first generation of new vortices, whose cross-stream locations in $y$ bracket the $y$-location (i.e. $y=0$ ) of the initial perturbing vortex. $E_{k, z}$ increases at a nearly uniform rate.

c) self replication: Each new vortex produces a set of independent critical layers, which undergo in turn the steps $a, b$. The self-replicating mechanism is triggered and a large number of vortices is rapidly generated. $E_{k, z}$ experiences a rapid increase.

d) zombie turbulence: The flow reaches a quasi-stationary state, i.e. $E_{k, z}$ is almost constant, $u_{z}$ shows strong and rapidly varying features but the critical layer structure is now barely visible. Reminiscences of "zombie" anti-cyclones (cyclones) and not-rolled up cyclonic (anti-cyclonic) vortex sheets are still visible in the $\omega_{z}$ field, but strong small-scale features are now ubiquitous. 


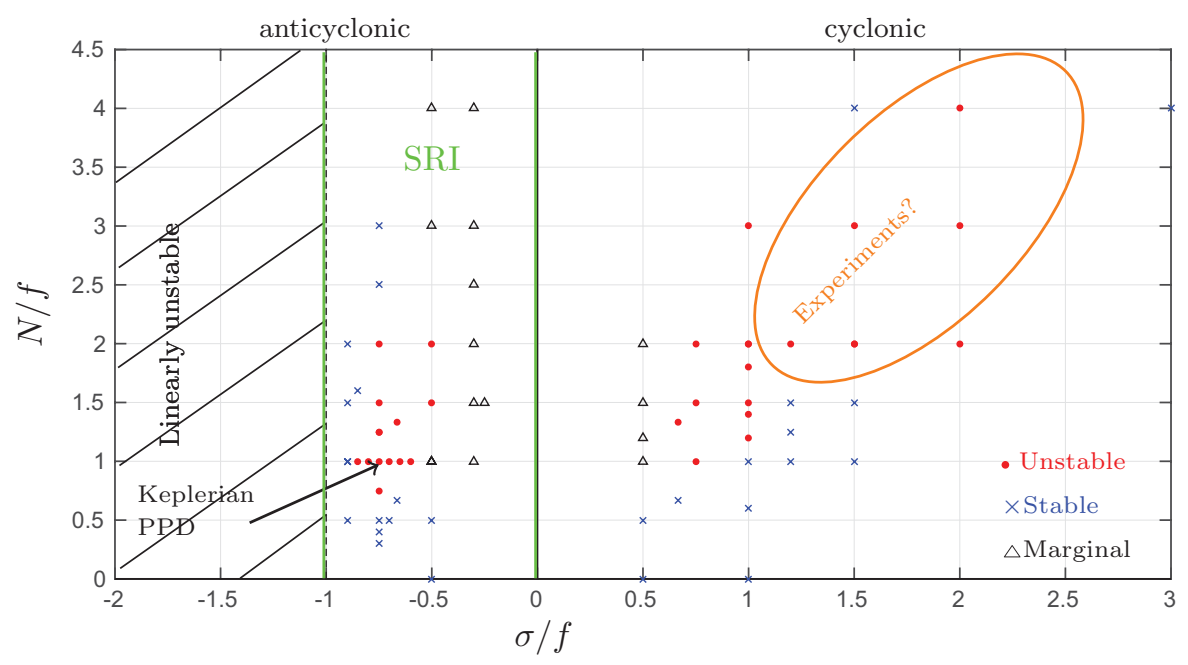

Fig. 3. Inviscid parameter map for ZVI. Each run is labelled with a red dot when unstable, a black triangle when marginal and a blue cross when stable. Vertical axis corresponds to $N / f$ while horizontal axis corresponds to $\sigma / f$. The left part of the diagram refers to anti-cyclonic cases, the right part to the cyclonic ones.

We originally (Marcus et al. 2013) perturbed the stratified TC equilibrium with an isolated, small, non-equilibrium vortex to produce ZVI and zombie turbulence. This was done to test numerically an analytical expression for the distance between a locally-confined initial perturbation and the location of the critical layer that was excited by the perturbation. However, other perturbations such as Kolmogorov noise and waves were also shown (Marcus et al. 2016) to produce zombie turbulence. It was found that if a flow produced zombie turbulence, the statisticallysteady properties of the late-time flow such as the energy spectrum as a function of wave number, were independent of the initial perturbation. A question of some importance here is: Does the form and amplitude of the initial perturbation have any significant effect on the regions in $\sigma / f-N / f-$ Re space where zombie turbulence can be produced? The answer to that question is beyond the scope of this study, but is answered in Wang et al. (submitted 2016): the form of the initial perturbation is nearly irrelevant as long as its amplitude (as measured by its Rossby number) is sufficiently large. In constructing the stability diagram (Fig. 3) we alternatively used different initial perturbations: isolated vortex, weak Kolmogorov turbulence and the zombie turbulence that was produced by a flow with slightly different values of the non-dimensional parameters.

\subsection{Inviscid stability diagram}

We explored the behavior of our flow varying the dimensionless parameters $\sigma / f$ and $N / f$ and label each case as stable, unstable or marginal, according to the 
protocol below:

- unstable: The flow undergoes all the stages listed in the previous section and in the end zombie turbulence appears and persists; the vertical kinetic energy rapidly oscillates around a large constant value.

- stable: Critical layers still show up, but at some point their strength starts decreasing and finally vanishes. The flow comes to the unperturbed state and the vertical kinetic energy relaxes to zero.

- marginal: The route to ZVI stops at an intermediate stage. All the velocity and vorticity fields appear like frozen, or evolve extremely slowly. The vertical kinetic energy saturates to a finite, slightly oscillating value.

The parameter $\sigma / f$ corresponds to the strain rate in TC geometry (Yavneh et al. $2001)$, while $N / f=1 /(2 F r)$ is half the inverse of the Froude number. The choice of the dimensionless parameters is not unique, but in the case of protoplanetary disk, rotation is the most persistent parameter and thus the best candidate to make the stability diagram non-dimensional. The results are reported in Figure 3. Depending on the sign of $\sigma / f$ we distinguish between anti-cyclonic flow (left) and cyclonic flow (right). Firstly we observe that the region $\sigma / f<-1$ is linearly unstable, which was observed in numerical simulations and confirmed by spectral analysis. This limit can be seen as the Rayleigh criterion in the plane Couette geometry. Instability occurs in two distinct zones. The first one spans quite a narrow region around the benchmark case of a Keplerian PPD, i.e. the same as Marcus et al. (2013) and Marcus et al. (2015), and is bounded from all sides. The second unstable zone belongs to the cyclonic region and looks analogous to the previous one except at large values of $\sigma / f$ and $N / f$, where no boundaries could be found. Approaching $\sigma=0$, the flow appears marginally stable, while at any other boundary the transition is always between stable and unstable cases, or at least no marginally stable case could be detected. The lack of instability at low $N / f$ and $|\sigma / f|$ suggests that whenever $N$ or $\sigma$ vanishes, ZVI does not appear. The existence of boundaries at large $N / f$ and moderate $|\sigma / f|$ indicated that ZVI disappears also for vanishing $f$, which was confirmed by numerical simulations (not reported here) at $f=0$. Thus we claim that ZVI may happen only when stratification, rotation and shear are of the same order, with the exception of the limit $f \rightarrow 0$ and $N / \sigma \sim 1$ where further studies are required. We note that a stability diagram like Figure 3 can be constructed for the SRI instability criterion for plane Couette flows. The green lines (Fig. 3) show the boundaries between which SRI exists.

\subsection{Viscous flow}

In the previous section we look at the stability diagram of ZVI in the inviscid limit. Here we discuss what happens when viscosity is considered. With regard to Equation (2.1), viscous dissipation, i.e. $\nu \nabla^{2} \mathbf{u}$, is now included and the Reynolds 
number $R e=L_{y}^{2} \sigma / \nu$ is introduced, where $L_{y}$ is the domain size length in the crossstream direction. We select two sample cases $N / f=1, \sigma / f=-0.75$ and $N / f=$ $2, \sigma / f=0.75$, and observe that ZVI occurs in viscous flows. The critical Reynolds number is around $R e_{c} \sim 5 \cdot 10^{6}$ for the anti-cyclonic case and $R e_{c} \sim 7.5 \cdot 10^{6}$ for the cyclonic case. We verify that these thresholds are not sensitive to the initial conditions and that we obtain similar results when considering viscosity only at the late stage, i.e. when turning on viscosity only once ZVI is fully developed. When $R e$ is large, i.e. for $R e>R e_{c}$, hyper-viscosity must be included beside viscosity. This happens because reasonable simulations times limit us to a space resolution at which the turbulent Kolmogorov scale is not resolved. Thus we adopt the same protocol as in the inviscid case and verify that results barely change for different types of hyper-viscosity. In Figure 2 (right) we report the time evolution of the maximum value of the vertical velocity $u_{z}$ observed inside the critical layers for different values of $R e$. One clearly sees that $u_{z}$ always increases with a similar slope at the beginning, showing that critical layers do form for any $R e$. When $R e<R e_{c}$, $u_{z}$ finally decreases at some time, showing the crucial role of critical layers when viscous dissipation is on. The critical Re number we find may appear unusually large (i.e. compared to other finite-amplitude instabilities), but this is consistent with the small thickness of baroclinic critical layers, which are extremely sensitive to viscous dissipation. The definition of $R e$ given above contains the cross-stream length $L_{y}$, as it is customary with the plane Couette flow, and thus provides a numerical benchmark for direct comparison with experiments, where cross-stream direction is often imposed by the shearing device. Nevertheless the position of critical layers (Marcus et al. 2013, 2015; Umurhan et al. 2016; Lesur \& Latter 2016), does not depend on $L_{y}$. A different $R e$ number could then be defined as intrinsically related to the instability $R e_{\delta}=\delta^{2} \sigma / \nu$, where $\delta$ is the thickness of the critical layers. In the sample case considered here we find $\delta / L_{y} \sim 10^{-5}$ and $R e_{\delta_{c}} \sim 35$.

\section{Conclusions}

We consider a rotating, stratified, plane Couette flow and perform direct numerical simulations in a triply periodic domain, using the shearing-sheet approximation. We extended previous works on ZVI (Marcus et al. 2013, 2015) constructing the stability diagram in the space $\sigma / f-N / f$. We find that the ZVI does occur in both cyclonic and anti-cyclonic regimes when the three ambient frequencies $f, \sigma, N$ are of the same order of magnitude. SRI may coexist with ZVI in the anti-cyclonic region and the flow is linearly unstable whenever $\sigma / f<-1$, which could serve as a first comparison in experiments. Looking for ZVI, the cyclonic region seems to be more suitable, not only because the unstable region is bigger, but also because SRI does not appear here. We do observe ZVI both in the inviscid limit and when viscosity is included. Numerical issues impose the implementation of hyperviscosity in any case, which may question the genuine character of our results. With regard to that we stress that independent simulations were performed with the finite-volumes code Athena (Marcus et al. 2015), where no hyper-viscosity was 
included. Also, as a general remark we observe that hyper-viscosity does dissipate energy at small-scales and ZVI originates from critical layers whose typical size is small, thus hyper-viscosity may, in principle, limit the onset of ZVI. Conversely, there is no clue to think that it may enhance ZVI in a fictitious way. Preliminary investigations show a large critical Reynolds number of the order $R e_{c} \sim 5 \cdot 10^{6}$, which follows from the small size of critical layers. Such a value can only be reproduced with very large experimental apparatus (typically $1.5 \mathrm{~m}$ in diameter). But an intermediate step in exploring ZVI, using more standard set-up, may be to excite baroclinic critical layers as previously achieved by Boulanger et al. (2008) in the related context of an inclined vortex in a linear stratification. This is encouraged by the fact that in our simulations critical layers always show up (at least the first generations), even for a Reynolds number orders of magnitude smaller than $R e_{c}$.

This work has been carried out thanks to the support of the A*MIDEX grant (ANR-11IDEX-0001-02) funded by the French Government "Investissements d'Avenir" program. P.S.M. is supported by NSF grants AST-1009907 and AST-1510703 and by NASA PATM grants NNX10AB93G and NNX13AG56G. Support for computational work comes from NSF XSEDE (NSF OCI-1053575) and NASA-HEC.

\section{References}

Barker, A.J., \& Latter, H.N., 2015, Mon. Not. R. Astron. Soc., 450(1), 21

Barranco, J.A., \& Marcus, P.S., 2005, Astrophys. J., 623(2), 1157

Barranco, J.A., \& Marcus, P.S., 2006, J. Comput. Phys., 219(1), 21

Boulanger, N., Meunier, P., \& Le Dizès, S., 2008, J. Fluid Mech., 596, 1

Chandrasekhar, S., 1960, Pro. Nat. Acad. Sci. U. St. A., 46(1), 141

Dubrulle, B., Marié, L., Normand, C., Richard, D., Hersant, F., \& Zahn, J.-P., 2005, J. Astron. Astrophys., 429, 1

Gole, D., Simon, J.B., Lubow, S.H., \& Armitage, P.J., 2016, Astrophys. J., 826(1), 18

Hawley, J.F., \& Balbus, S.A., 1991, Astrophys. J., 376, 223

Le Bars, M., \& Le Gal, P., 2007, Phys. Rev. Lett., 99, 064502

Lesur, G., Kunz, M.W., \& Fromang, S., 2014, J. Astron. Astrophys., 566, A56

Lesur, G.R.J., \& Latter, H., 2016, Mon. Not. R. Astron. Soc., 462(4), 4549

Marcus, P.S., Pei, S., Jiang, C.-H., Barranco, J.A., Hassanzadeh, P., \& Lecoanet, D., 2015, Astrophys. J., 808(1), 87

Marcus, P.S., Pei, S., Jiang, C.-H., \& Hassanzadeh, P., 2013, Phys. Rev. Lett., 111, 084501

Molemaker, M.J., McWilliams, J.C., \& Yavneh, I., 2001, Phys. Rev. Lett., 86, 5270

Nelson, R.P., Gressel, O., \& Umurhan, O.M., 2013, MNRAS, 435, 2610

Rayleigh, L., 1917, Pro. R. Soc. London A: Math. Phys. Eng. Sci., 93(648), 148

Shalybkov, D., \& Rüdiger, G., 2005, Astron. Astrophys., 438(2), 411

Umurhan, O.M., Shariff, K., \& Cuzzi, J.N., 2016, Astrophys. J., 830(2), 95

Wang, M., Pei, S., Jiang, C.-H., Facchini, G., \& Marcus, P.S., J. Fluid Mech.

Yavneh, I., McWilliams, J.C., \& Molemaker, M.J., 2001, J. Fluid Mech., 448, 1 


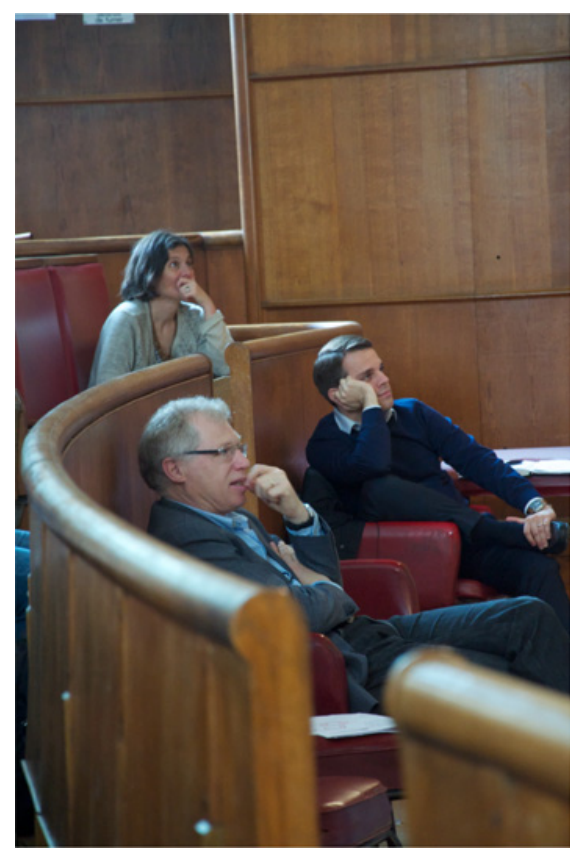

Bérengère Dubrulle, Michel Rieutord and Stéphane Mathis

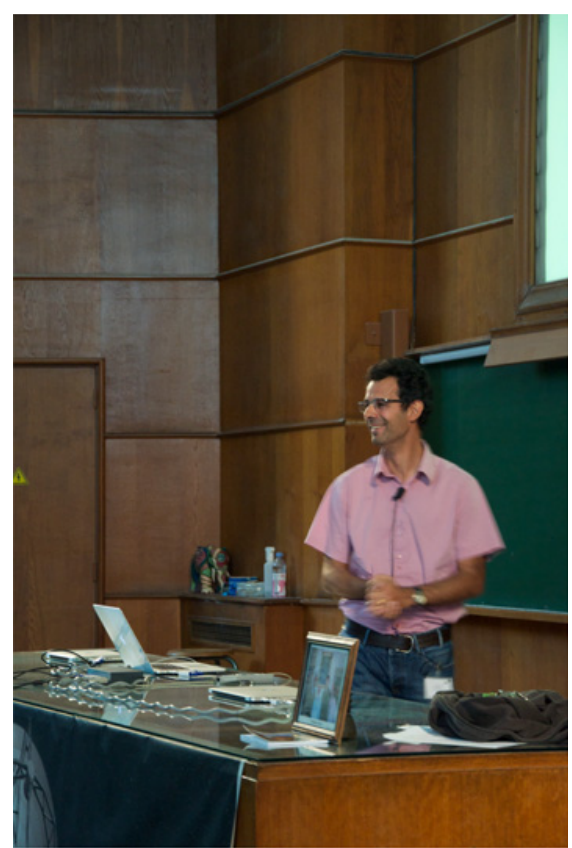

Sébastien Fromang 